\title{
Comportamiento de la linea naso-frontal para la determinación de clases en vicuñas (Vicugna vicugna)
}

\author{
Behavior of the naso-frontal line for determining classes vicuñas (Vicugna vicugna)
}

\author{
Marco Zúñiga ${ }^{1}$ Norma Bujaico ${ }^{2}$
}

http://dx.doi.org/10.21503/CienciayDesarrollo.2014.v17i2.04

\section{RESUMEN}

Se propone un método de observación directa para identificar las clases de animales que nos acerquen a determinar una edad aproximada, tomando como indicador una línea imaginaria a nivel de la frente y la nariz (Naso - Frontal), siendo un perfil hendido muy apreciable en las crías, un poco más recto en las juveniles, línea casi recta en adultos y la curvatura hacia fuera de la parte de la nariz en los seniles asemejando a un pico de ave.

Palabras Clave: Vicuñas crías, juveniles, adultas, seniles.

\section{ABSTRACT}

Direct observation method is proposed to identify the types of animals that bring us determine an approximate age, taking as an indicator an imaginary line on the forehead and nose (Naso - Front), being a very significant cleft profile in young, a little straighter in youth, adults almost straight line and curve out from the side of the nose senile resembling a beak.

Keywords: Vicuña young, youth, adult, senile.

\section{INTRODUCCIÓN}

La vicuña es un mamífero perteneciente a la familia camelidae cuya historia evolutiva empezó hace aproximadamente unos 40 millones de años en América del Norte.

De este género según investigaciones, surgieron las cuatro especies de camélidos sudamericanos que actualmente conocemos: La llama y la alpaca como camélidos domésticos, la vicuña y el guanaco como silvestres.

La vicuña (Vicugna vicugna) es el más pequeño, grácil, escaso y fino ejemplar dentro de esta familia, que habita en la Cordillera de los Andes de Ecuador, Perú, Bolivia, Argentina y Chile entre los 3,800 a 4,800 metros de altitud y cuya importancia radica especialmente en la finura y calidad de su fibra.

\footnotetext{
1 Médico Veterinario, Zootecnista. Asesor del Comité de Uso Sustentable de la Vicuña del CIPTT Tullpacancha de la Universidad Alas Peruanas. Asesor de la Comunidad Campesina de Lucanas, Pampa Galeras y otras. E-mail: Anzuv20@hotmail.com

2 Ingeniero zootecnista, Universidad Nacional de Huancavelica. E-mail: normisbm15@hotmail.com
} 
A partir del año 1994, esta especie es sometida a un manejo intenso tanto en su estado silvestre como aquellas poblaciones que se encuentran en semi cautiverio mediante el manejo en cercos permanentes, las cuales primeramente se traducen en conteos comunales, capturas, esquilas, traslados con fines de repoblamiento y finalmente el control y vigilancia de las mismas.

Para todo lo descrito anteriormente, es imprescindible tener conocimiento de algunas caracteristicas naso frontales en las vicuñas, que nos acerquen a determinar una edad aproximada del animal de manera práctica, y de tal forma poderlas agrupar en: vicuñas crías, juveniles, adultas y seniles, sin necesidad de boquear ni estresar por captura al animal, para practicar exámenes tediosos que nos indiquen su edad.
Existe una línea imaginaria a nivel de la frente y la nariz de las vicuñas denominada naso-frontal la cual determina el perfil de las mismas y de acuerdo al comportamiento de ésta se puede determinar la clase y edad aproximada.

Quede pues en claro que el objetivo de este trabajo es contribuir a determinar en el tiempo más corto, la clases en vicuñas a partir de una simple vista al animal y agruparlas en una tabla cronológica que nos permita tomar nota de ellas en actividades propias de esta especie sin temor a caer en gruesos errores de clasificación, como los que se incurre en el momento de hacer censos o apuntes en cuanto al llenado de los registros de captura y esquila de estos camélidos silvestres.

\section{MATERIALES Y MÉTODOS}

Para la realización del presente, se trabajó una muestra a nivel nacional de 840 vicuñas (2013), a las que se les hizo la medición de la hendidura naso-frontal o perfil, en los sitios y cantidades que a continuación se detalla, correspondiente a las campañas de captura y esquila del año 2013:

\begin{tabular}{lcrrrrr}
\multicolumn{1}{c}{ REGIÓN } & LUGAR & CRÍAS & JUVENILES & ADULTOS & SENILES & TOTAL \\
Huancavelica & Tullpacancha & 46 & 25 & 53 & 11 & 135 \\
Ayacucho & Pampa Galeras & 159 & 108 & 244 & 61 & 572 \\
Arequipa & Colca Huallata & 25 & 31 & 33 & 08 & 97 \\
Cusco & Torre Estrella & 12 & 10 & 11 & 03 & 36 \\
TOTAL & & $\mathbf{2 4 2}$ & $\mathbf{1 7 4}$ & $\mathbf{3 4 1}$ & $\mathbf{8 3}$ & $\mathbf{8 4 0}$ \\
\hline
\end{tabular}

Fuente: Autor, 2014

Debido a la carencia de dimorfismo sexual en las vicuñas, solamente se pueden identificar a éstas según su comportamiento, siendo importante definir las clases de vicuñas para un mejor entendimiento del presente trabajo:
Vicuñas crías. Ejemplares de ambos sexos cuya edad está enmarcada desde su nacimiento hasta antes de los 12 meses de edad. (Figura 3)

Vicuñas juveniles. En las hembras se las 
considera desde los 12 meses de edad o su primer año de vida hasta la parición de su primera cría, y los machos entre los 12 meses de edad y su salida de la tropilla juvenil. (Figura 4)

Vicuñas adultas. Son las hembras después de su primer parto hasta la parición de su séptima u octava cría, coincidiendo con unos 10 a 11 años de edad. En cuanto a los machos adultos se consideran como tal desde la salida de la tropilla juvenil hasta su expulsión del grupo familiar. (Figura 5)

Vicuñas seniles. A las hembras se les considera viejas desde su término de vida fértil hasta su muerte y en machos desde la salida de su grupo familiar hasta su muerte, teniendo una edad aproximada de 12 años de edad. (Figura 6)

El método utilizado para este trabajo fue el de la observación directa de las vicuñas en los lugares de captura y su posterior medición de la hendidura naso - frontal con relación a la línea recta trazada desde la frente hasta la punta de la nariz.

\section{RESULTADOS}

Para obtener conclusiones respecto a la estructura de la población, es indispensable diferenciar las vicuñas con relación a su edad para evitar someter a estres por capturas al animal, aprovechando mas bien la esquila para hacer una clasificación minuciosa.
En cuanto a resultados se tiene lo siguiente:

Se registra un perfil hendido muy apreciable en las crías $(\mathrm{x}=-2,16$ figura 3$)$, un poco más recto en las juveniles $(x=-1,28$ figura 4$)$, línea casi recta en adultos ( $x=-0,34$ figura 5$)$ y la curvatura hacia fuera de la parte de la nariz en los seniles $(x=$ $+0,72$ figura 6) asemejando a un pico de ave.

\section{DISCUSIÓN}

Para determinar las clases en vicuñas, solamente se tiene que observar con detenimiento el perfil de la cabeza del animal y tomar nota principalmente de la hendidura naso -frontal corroborando en esta tarea la morfología del cuerpo del animal. Claro está que un cuerpo pequeño y una cabeza con una hendidura naso - frontal pronunciada corresponde inequívocamente a una cría y una vicuña con un cuerpo desarrollado pero con una nariz en forma de pico de ave, será de una vicuña senil.

Tratándose de una especie silvestre, es difícil su manipulación por eso, este trabajo contribuye a que mediante la observación directa se determine las clases en vicuñas cuyo objetivo fundamental es no caer en confusiones ni equívocos en el momento de hacer censos o conteos poblacionales, apuntes en registros de captura y esquilas, seleccionar vicuñas con fines de traslado o repoblamiento y a la vez identificar cronológicamente los cadáveres de estos animales encontrados a nivel de campo. 


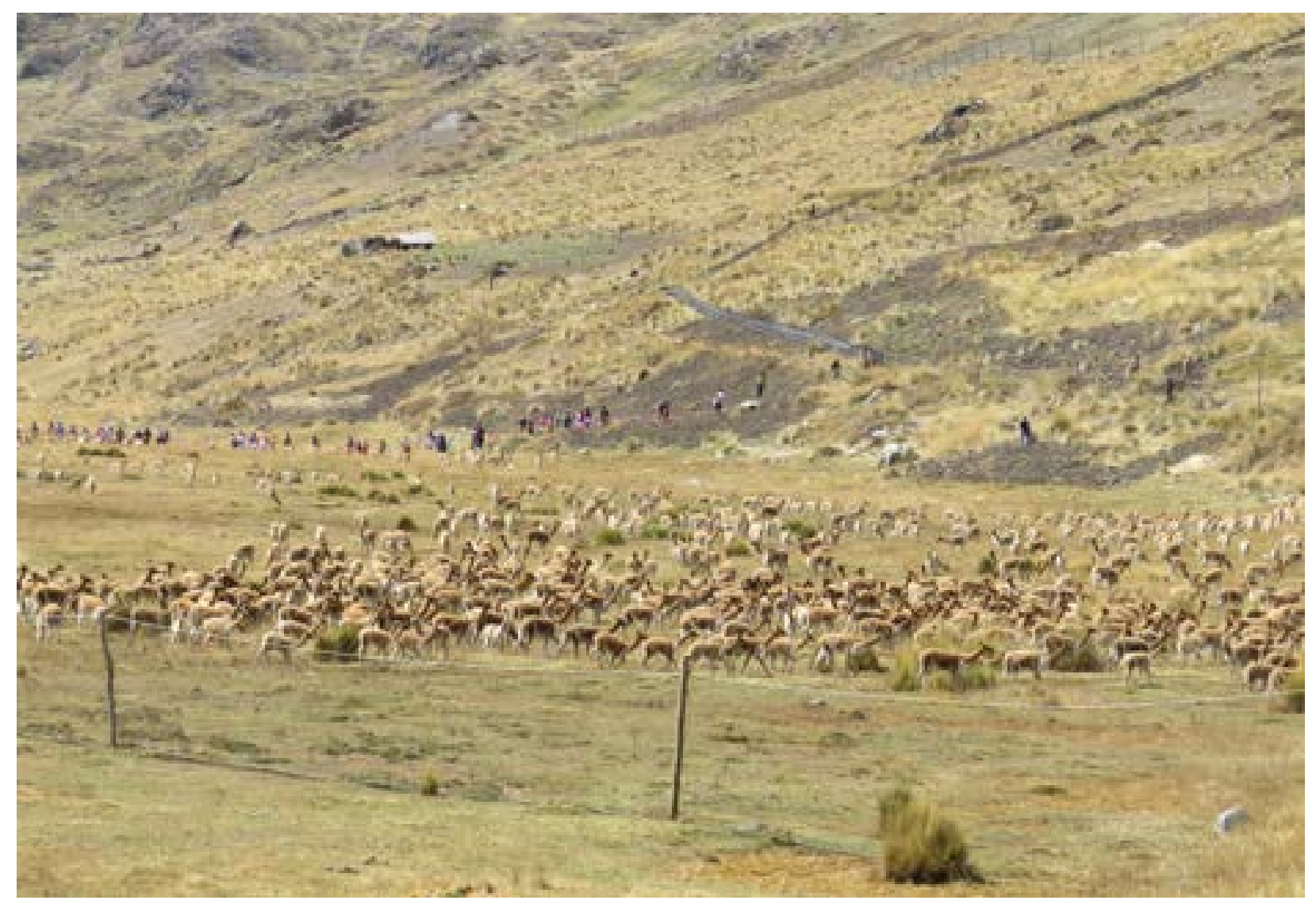

Figura 1. Chacco 2014, Centro de Investigación CIPTT Tullpacancha, Churcampa, Huancavelica, Universidad Alas Peruanas.

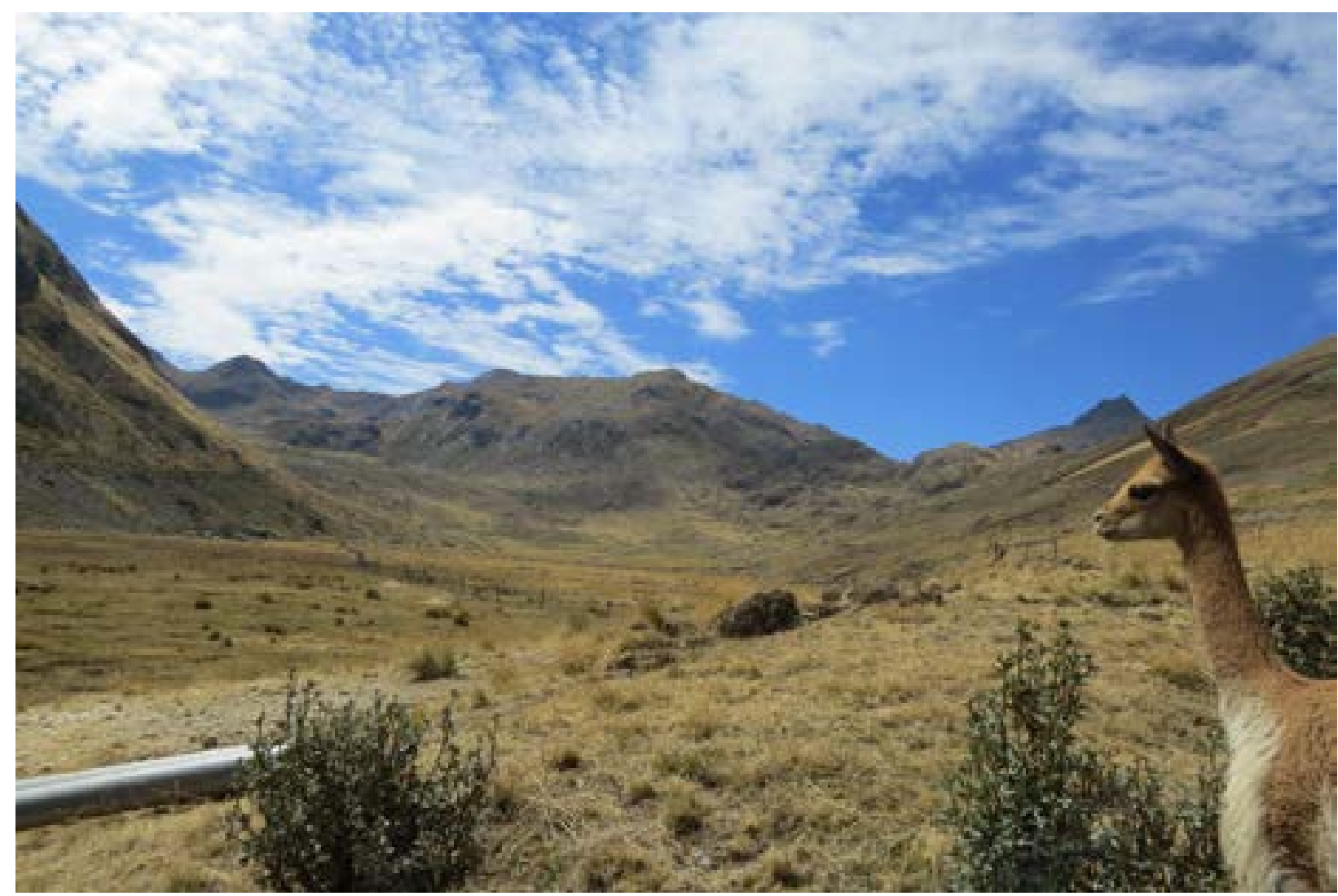

Figura 2. Vicuña macho adulto. Centro de Investigación CIPTT Tullpacancha, Churcampa, Huancavelica, UAP. 
Figura 3.

VICUÑAS CRÍAS
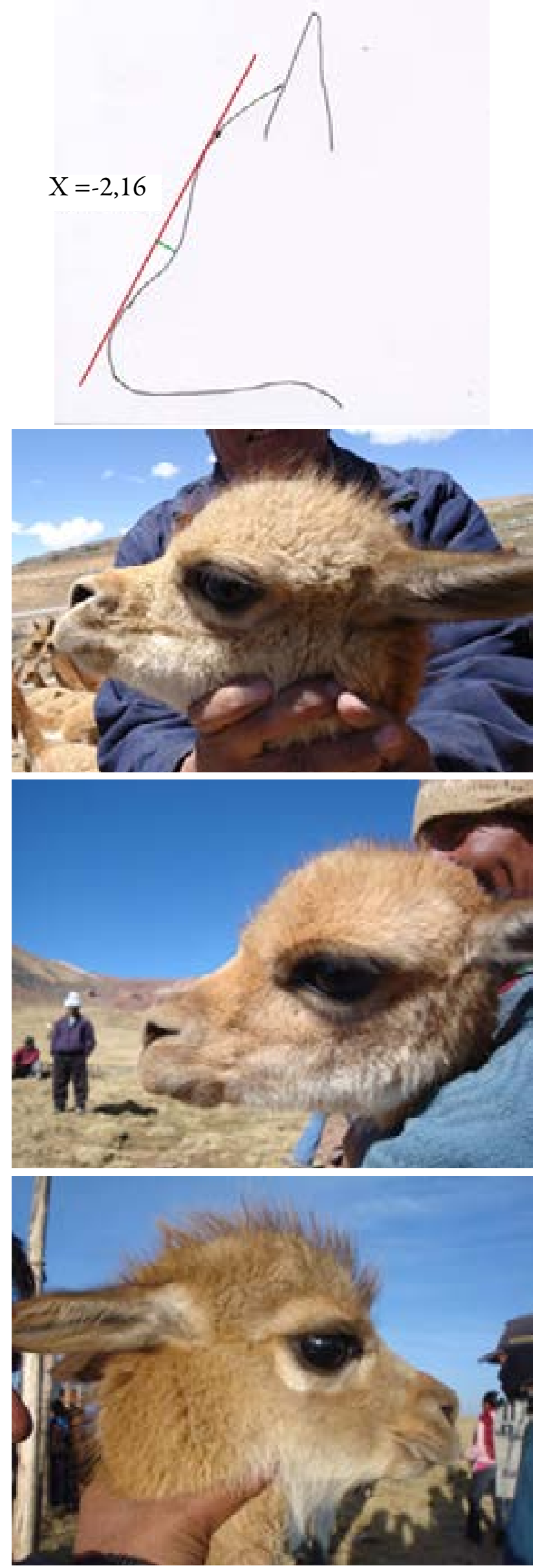

Ciencia y Desarrollo 17 (2), julio-diciembre 2014. 39:47 V. E.: ISSN 2409-2045 
Figura 4.

VICUÑAS JUVENILES
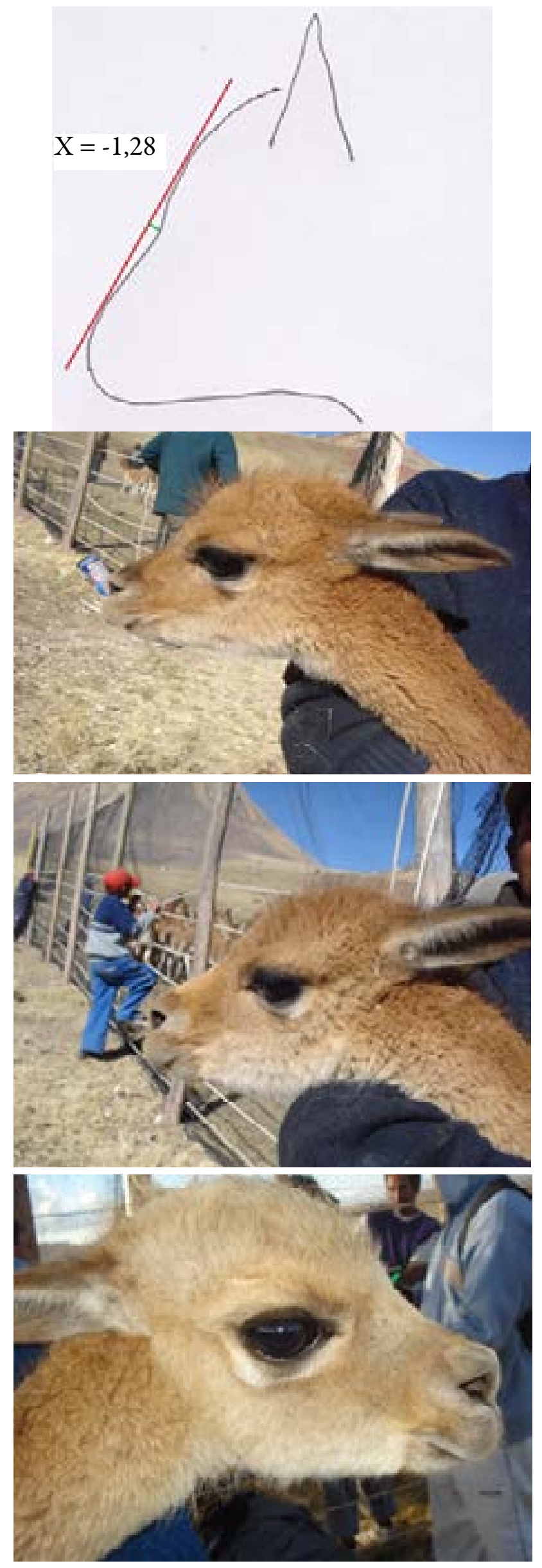
Figura 5.

VICUÑAS ADULTAS
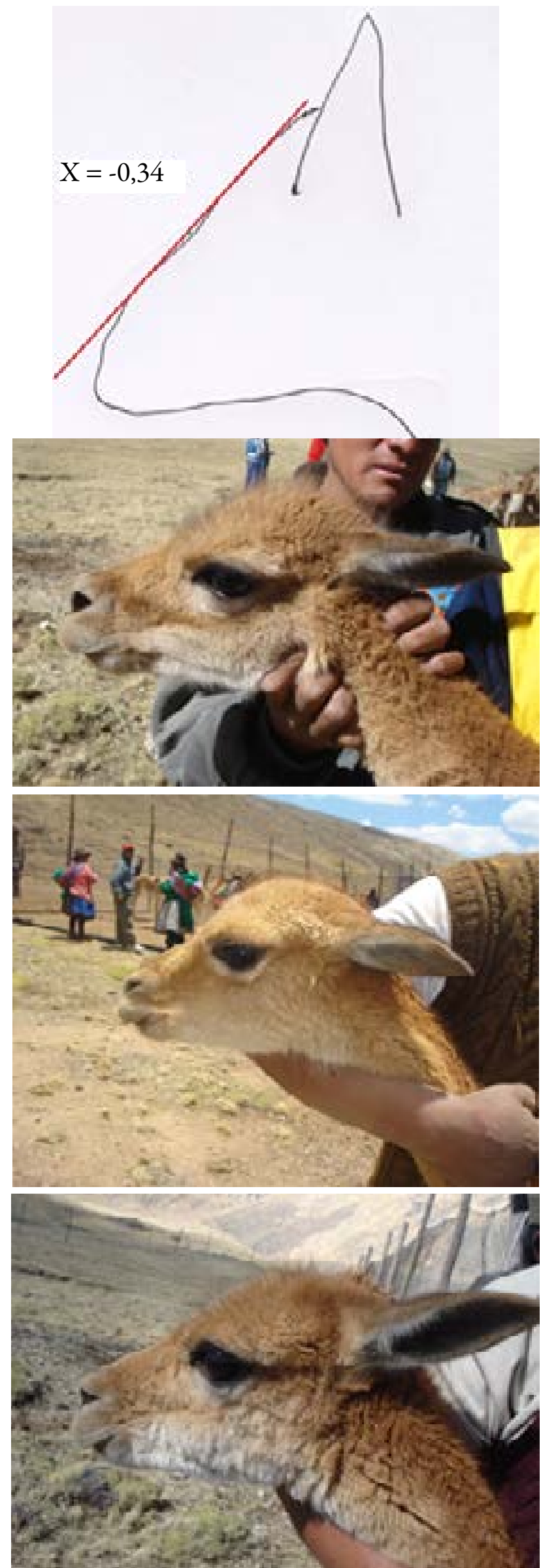

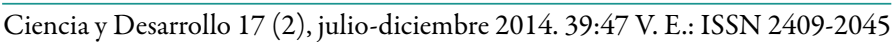


Figura 6.

VICUÑAS SENILES
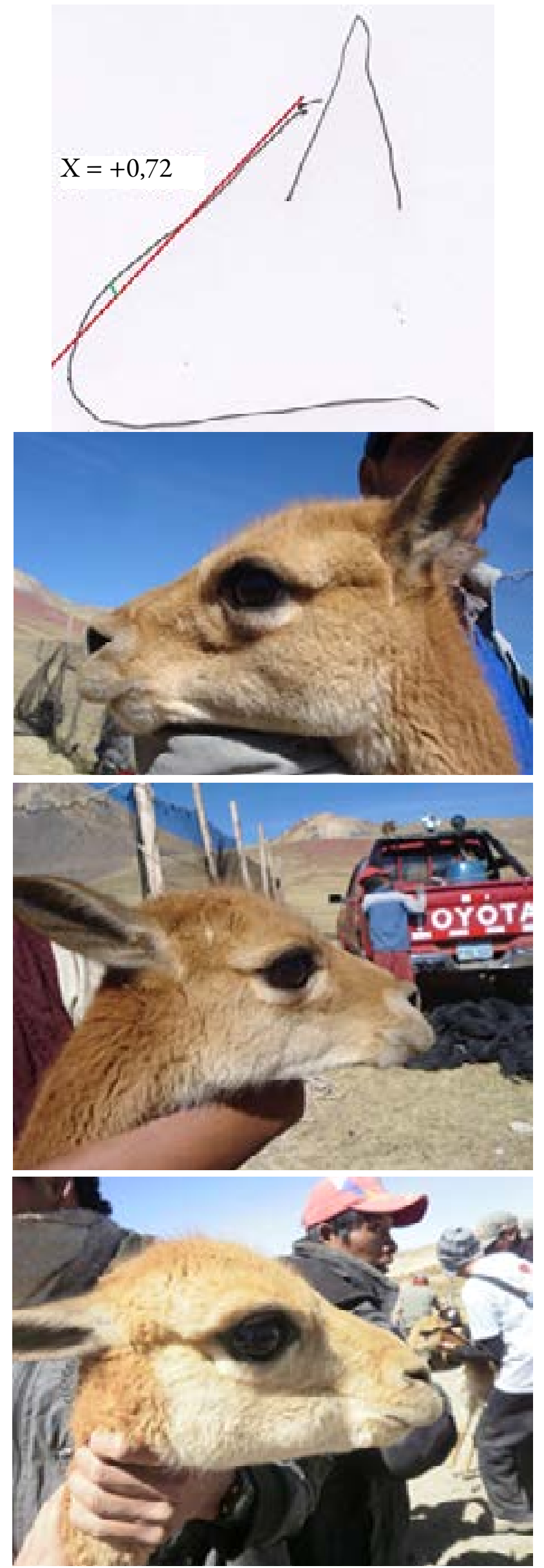


\section{BIBLIOGRAFÍA}

1. Bonavia, D. 1996. Los camélidos sudamericanos (Una introducción a su estudio). Edit. Lluvia. Perú (Lima).

2. Brack, E. 1980. Informe del Proyecto Especial "Utilización racional de la Vicuña". Ministerio de Agricultura. Lima-Perú. Pág. 12.

3. Cajal, J. 1989. Uso del hábitad por vicuñas y guanacos en la Reserva San Guillermo, Argentina. Vida Silvestre Neotropical 2:21-31.

4. Cueto, L. ; Ponce, C.; Cardich, E.; Rios, M. 1985. El Manejo de la vicuña, su contribución al desarrollo rural en los altos andes del Perú. Guía FAO Conservación No 11. Organización de las Naciones Unidas para la Agricultura y la Alimentación. Roma.

5. Franklin, W. 1974. El comportamiento social de las vicuñas. IVITA.CICCS.CIID. Canadá.

6. Hoces, D. 1995. Promoción de los camélidos sudamericanos. En: Festival Internacional de Camélidos Sudamericanos. Lima.

7. Hofmann, R.; Otte, K.; Ponce, M.; Rios, M. 1983. El manejo de vicuña silvestre. Tomo I y II. Publicado por Deutsch Gesellsharft fur Technische Zusammenarbeit (GTZ) GMBH. Sociedad Alemana de Cooperación Técnica. Eschborn.
8. Puig, S. y Videla, F. 2000. Dinámica poblacional y uso del habitad por el guanaco Seminario Manejo Sustentable de la Vicuña y el Guanaco. Santiago, Chile.

9. Sánchez, E. y Yaringano, R. 1987. Manejo de vicuñas. Primera aproximación al desarrollo de un modelo de manejo. Ministerio de Agricultura. Proyecto Especial "Utilización Racional de la Vicuña”. Lima.

10. Torres, H. 1983. Distribución y conservación de la vicuña (Vicugna vicugna). Grupo especialista en Camélidos Silvestres Sudamericanos, Comisión de sobrevivencia de Especies. Unión Internacional para la conservación de la Naturaleza y sus Recursos. Informe especial No 1 Julio 1983. Suiza.

11. Zarate, J. y Carcausto, J. 1994. Evaluación del comportamiento social de la vicuña, en el centro de conservación Kala Kala. UNA. Facultad de ciencias Biológicas. Área de Ecología-Puno.

12. Zúñiga, Marcos. 2007 La vicuña y su manejo técnico. Edit. Fondo Editorial de la Universidad Alas Peruanas,. 166 pp. LimaPerú.

13. Zúñiga, Marcos. 2014 La fibra de vicuña y su trabajo en talleres de clasificación. Edit. Fondo Editorial de la Universidad Alas Peruanas, 170pp. Lima. 